\title{
Absorption of prednisolone in patients with Crohn's disease
}

\author{
J A SHAFFER. S E WILLIAMS. L A TURNBERG, ${ }^{*}$ \\ J B HOUSTON, AND M ROWLAND
}

From the Department of Medicine, Hope Hospital, and Department of Pharmacy, University of Manchester, Manchester

SUMMARY The absorption of prednisolone in patients with Crohn's disease was investigated. Seven patients with Crohn's disease and eight normal control subjects were given a tracer dose of tritiated prednisolone with $20 \mathrm{mg}$ cold prednisolone by mouth. On a separate occasion they were given an intravenous injection of radiolabelled prednisolone. After oral ingestion only $53.4 \pm 11.7 \%$ of labelled material was excreted in the urine of Crohn's patients compared with $82.5 \pm 3.6 \%$ in the normal subjects. The oral/intravenous availability ratio was $0.61 \pm 0.14$ in Crohn's patients and $0.89 \pm 0.07$ in the normal group. Areas under plasma concentration-time curves were lower in patients than normal subjects and the oral/intravenous ratios were $0 \cdot 6 \pm 0 \cdot 2$ and $0.86 \pm 0.09$ respectively. Faecal excretion of radioactivity after oral ingestion was greater in Crohn's patients $(19 \cdot 3 \pm 2 \cdot 5 \%, n=3)$ than in normal subjects $(7 \pm 2 \cdot 8 \%, n=4)$. The range for each type of measurement was much wider in the patient group than in the normal subjects. These data suggest that patients with Crohn's disease do not absorb prednisolone normally and that absorption varies between patients.

Corticosteroids are commonly used in the treatment of patients with Crohn's disease but clinical responses are variable and the development of side-effects unpredictable.' Several factors may contribute to this variability. Thus absorption from the gut, or metabolism and elimination may not be uniform, or there may be an intrinsic variability in responsiveness of the disease. The present studies were performed to assess the possibility that absorption of prednisolone may be impaired and thus contribute to the variable responsiveness of patients with Crohn's disease.

\section{Methods}

\section{SUBJECTS}

Seven patients with Crohn's disease (two men and five women) aged 22-50 years were studied. The diagnosis of Crohn's disease was based on generally accepted clinical, radiological, and histological

* Address for reprints: Professor L A Turnberg. Department of Medicine. (University of Manchester School of Medicine). Hope Hospital. Eccles Old Road. Salford M6 8HD.

Received for publication 17 May 1982 criteria. Disease activity was mild to moderate (Crohn's Disease Activity Index, ${ }^{2}$ ranged from 81-232, mean $150 \cdot 7$ ) and all patients were attending the outpatient clinic. Macroscopic evidence of disease was present in the ileum alone in two patients, one had gastric plus ileal involvement, three had an ileocolitis, and one had colonic disease only. One patient had had a right hemicolectomy and one, an ileectomy. All patients were taking sulphasalazine at the time of the study and five patients prednisolone (dose range 10-30 mg). In addition, one patient each was taking azothiaprine, cimetidine, or loperamide. Only the patient taking $30 \mathrm{mg}$ prednisolone had clinically observable corticosteroid side-effects - for example, moon face. No patient had steatorrhoea or a protein-losing enteropathy.

Eight healthy volunteers (six men, two women, aged 20-22 years) served as control subjects. The protocol was reviewed by Salford Area Health Authority Ethical Committee and the volunteers gave their informed consent.

STUDY

Absorption of prednisolone was measured from the 
cumulative urinary excretion of radioactivity after oral administration of isotopically labelled prednisolone. This was compared with urinary excretion of isotope after intravenous administration of labelled drug, and an oral/intravenous ratio was calculated. Plasma appearance and disappearance curves were also constructed after faecal excretion of isotope after oral administration was determined.

After a 12-hour fast a $20 \mathrm{ml}$ solution (one part $95 \%$ ethanol to nine parts anise flavoured $20 \%$ sucrose) containing $20 \mathrm{mg}$ prednisolone and $30 \mu \mathrm{Ci}$ $\left[6,7-{ }^{3} \mathrm{H}\right]$-prednisolone $(50 \mathrm{Ci} / \mathrm{mmol})$ was administered orally and $5 \mathrm{ml}$ venous samples were collected into heparinised tubes at frequent intervals for 24 hours. A control urine sample had been collected on wakening and further collections were made at regular intervals for 60 hours. In four control subjects and three patients faecal samples were collected for five days.

On the intravenous study day $20 \mathrm{mg}$ 'cold' prednisolone was given orally followed one hour later by the intravenous administration of $30 \mu \mathrm{Ci}^{3} \mathrm{H}$ prednisolone solution (one part $95 \%$ ethanol to 19 parts normal saline). No faecal collections were made but otherwise the protocol was as for the oral study.

The precision of the radioactive dose administered was checked by determining the residual tritium in syringes and containers after drug administration.

Aliquots of plasma and urine were assayed in duplicate for radioactivity in RIA luma (LKB Wallac) using a LKB 1216 Rackbeta scintillation counter.

Faecal samples were homogenised in $200-300 \mathrm{ml}$ methanol in a Colsworth Stomacher. Five $1 \mathrm{ml}$ samples of each suspension were taken for the determination of radioactivity after treatment with Lumasolve (LKB-Wallac) and the addition of Lumagel (LKB-Wallac). Labelled prednisolone was obtained from the Radiochemical Centre, Amersham, and purity checked by high pressure liquid chromatography as described below.

\section{DATA TREATMENT}

Cumulative ${ }^{3} \mathrm{H}$ in urine and faeces was estimated by summing the radioactivity in each of the timed samples. The area under the plasma concentrationtime curve between zero and 24 hours was calculated by the trapezoidal rule. The area from 24 hours to infinity was obtained by dividing the 24 hour value by the rate constant associated with the terminal phase. The terminal half-life was obtained from semi-logarithmic plasma concentration-time and urinary excretion rate-time plots.
HIGH PRESSURE LIQUID CHROMATOGRAPHY

FRACTIONATION OF PLASMA RADIOACTIVITY

The plasma sample taken three hours after administration of the tracer dose was analysed by high pressure liquid chromatography. An aliquot of plasma was added to an equal volume of methanol containing an internal standard (triamcinolone) mixed and the precipitated protein centrifuged. The supernatant was injected on to a $200 \times 5 \mathrm{~mm}$ (internal diameter) stainless steel column packed with Spherisorb-5-MOS which was preceded by a $50 \times 5 \mathrm{~mm}$ (internal diameter) silica guard column. The mobile phase comprised $15 \%$ isopropranolol and $1 \%$ acetic acid in water and ultraviolet detection at $254 \mathrm{~mm}$ was used.

With the use of this method prednisolone was successfully resolved from prednisone and hydrocortisone. The eluant was collected every 30 seconds after injection, scintillation fluid (Lumagel) added, and radioactivity determined. Histograms were plotted of the radioactivity eluted with time and the percentage radioactivity associated with prednisolone was calculated.

\section{Results}

In the control group the percentage radioactivity recovered from the urine was $82.5 \pm 3.6 \%$ (mean \pm SD) after the oral study and $92.4 \pm 5.3 \%$ after the intravenous study giving a tritium availability ratio of $0.89 \pm 0.07$ (Fig. 1). In four subjects the faecal radioactivity recovered was $7 \pm 2.8 \%$ (mean $\pm S D)$ after oral administration of prednisolone.

In the Crohn's disease patients the urine radioactivity recovered was $53.4 \%( \pm 11.7 \%)$ after oral administration and $89.9 \%( \pm 4.2 \%)$ after intravenous administration. The tritium availability ratio was $0.61( \pm 0.14)$ (Fig. 1). The recovery after oral administration is significantly less than in the control group ( $p<0.001$, Student's $t$ test). In three patients cumulative radioactivity recovered in the faeces after oral ingestion was $19.3( \pm 2.5 \%)$.

The total area under the plasma tritium concentration-time curve between time zero and infinity, was $19,000 \mathrm{dpm} / \mathrm{ml} / \mathrm{h}( \pm 1500)$ after oral administration, and $22,000 \mathrm{dpm} / \mathrm{ml} / \mathrm{h}( \pm 1100)$ after the intravenous study in the control group (Fig. 2). Thus the oral/intravenous tritium area ratio is 0.86 $( \pm 0 \cdot 09)$. Corresponding values in the patient group were $12,100 \mathrm{dpm} / \mathrm{ml} / \mathrm{h}( \pm 3800)$ oral and 20,200 $\mathrm{dpm} / \mathrm{ml} / \mathrm{h}( \pm 2200)$ intravenous (Fig. 3). The oral/ intravenous ratio in this group is $0.60( \pm 0.20)$, which is significantly less than in the control group $(p<0.001)$.

High pressure liquid chromatography frac- 


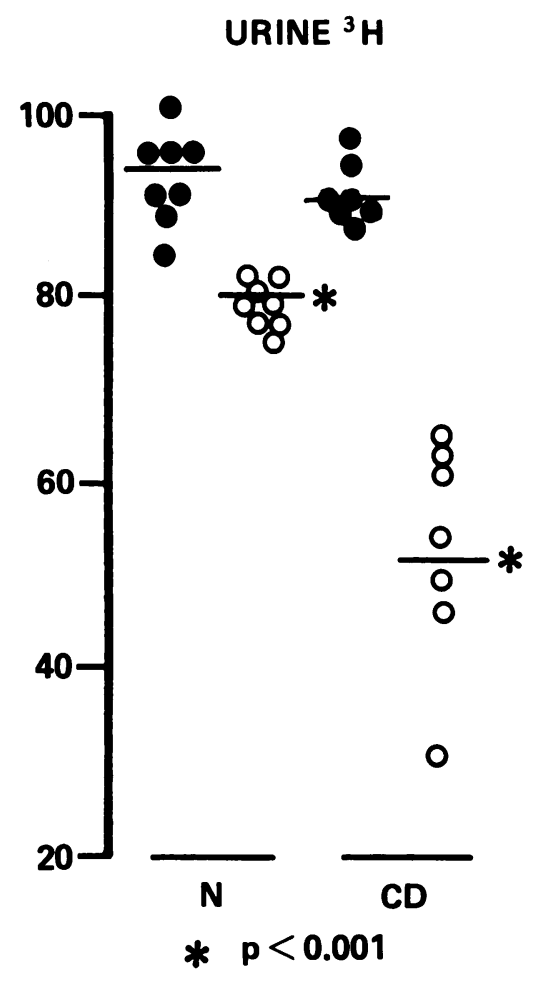

Fig. 1 Cumulative urinary tritium recovery in normal subjects and Crohn's disease patients after administration of $\left[6,7-{ }^{3} \mathrm{H}\right]$-prednisolone by oral $(\mathrm{O})$ and intravenous $(\bullet)$ routes. $(N=$ normal subjects; $C D=$ Crohn's disease. $)$

tionation of plasma radioactivity from samples taken three hours after administration of the tracer showed that in all subjects prednisolone accounted for at least $90 \%$ of the radiochemical species, and this was similar in both groups of subjects after either route of administration.

Tritium plasma concentration-time and urinary excretion rate-time curves allowed determination of half-lives. There was no significant difference between the half-lives obtained by the two methods in either subject group. The route of administration did not influence the results: normal subjects, intravenous $3.9 \mathrm{~h}( \pm 0.4)$ and oral $3.9 \mathrm{~h}( \pm 0.6)$, Crohn's disease patients, intravenous $3 \cdot 2 \mathrm{~h}( \pm 0 \cdot 1)$, and oral $4.0 \mathrm{~h}( \pm 1.0)$.

None of the subjects experienced any untoward effects from the study. There was no correlation between urinary excretion data or plasma concentration data, and either the severity of disease as indicated by the disease activity index or the anatomical site of the disease.

\section{Discussion}

Plasma concentrations of prednisolone vary considerably after oral ingestion of the same dose by normal subjects or patients. ${ }^{34}$ The source of this variability may lie in intersubject differences in either prednisolone disposition or in extent of absorption from the intestinal tract.

The protocol used in our studies involved the use of a tritiated prednisolone tracer. This allows the non-specific assay of all prednisolone-related chemical species simultaneously. Availability fractions based on urinary recovery of tritium represent the maximum possible estimate of prednisolone availability. The results obtained from urinary tritium recovery were very similar to our observations on plasma radioactivity, and high pressure liquid chromatography showed that this radioactivity was predominantly prednisolone, suggesting that the availability fractions reported here are reasonable estimates of the availability of prednisolone.

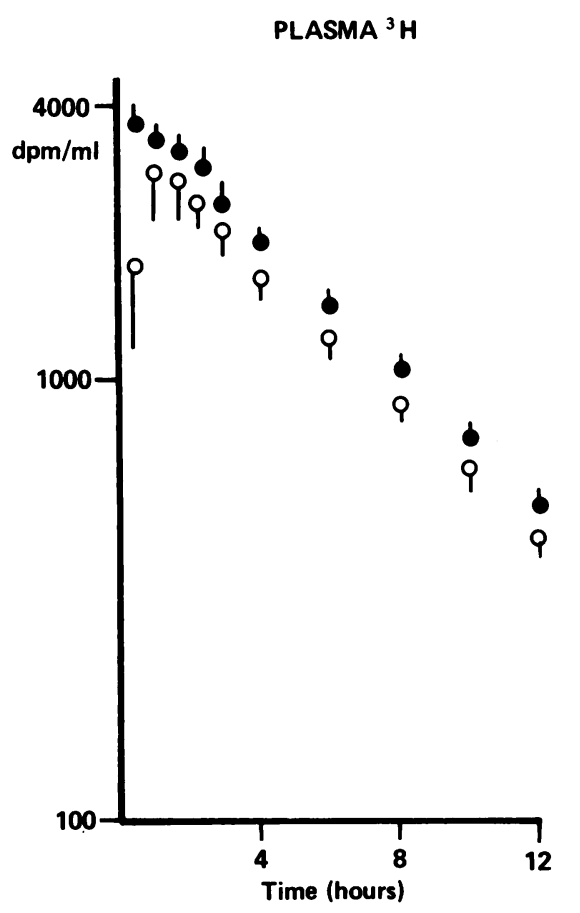

Fig. 2 Plasma concentration-time curves for tritium in normal subjects after administration of $\left[6,7-{ }^{3} \mathrm{H}\right]$ prednisolone by oral $(\circ)$ and intravenous $(\bullet)$ routes. Mean data with SD shown. 


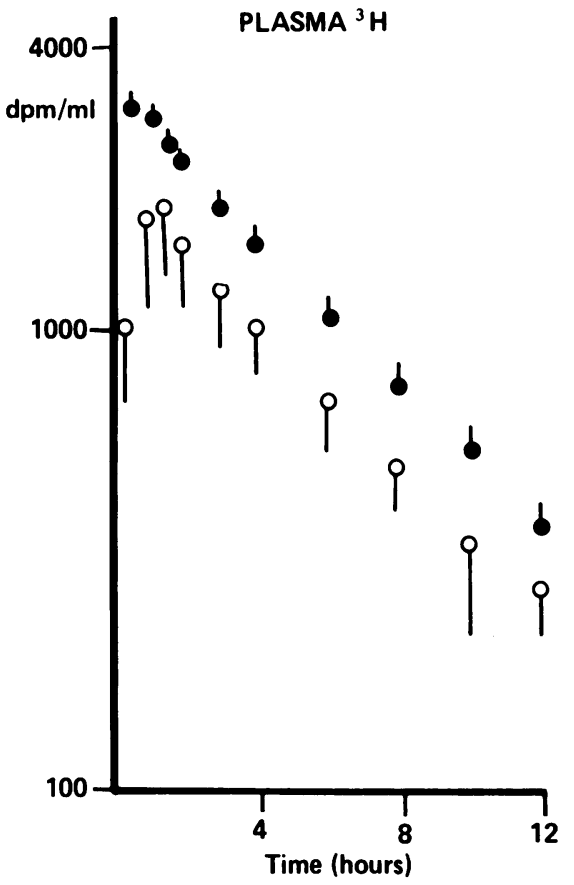

Fig. 3 Plasma concentration-time curves for tritium in Crohn's disease patients after administration of $\left[6,7 \cdot{ }^{3} \mathrm{H}\right]$ prednisolone by oral $(\circ)$ and intravenous $(\bullet)$ routes. Mean data with $S D$ shown.

The design of the present study allowed the tracer to be administered by both oral and intravenous routes. In the first case the tracer was incorporated with the cold dose in solution. Hence no problems with solid dosage form dissolution and mixing of cold and tritiated prednisolone should be encountered. In the second case the tracer was given intravenously one hour after the oral cold dose that is, at a time when the body load of steroid was near maximal. This design is advantageous, as the disposition of prednisolone is known to be nonlinear. ${ }^{56}$ Sources of this non-linearity include steroid binding to globulins, ${ }^{7}$ the interconversion between prednisolone and prednisone, ${ }^{8}$ and the renal clearance mechanisms for prednisolone. ${ }^{9}$ Such non-linear processes may well produce routedependent effects on pharmacokinetic calculations. In our study, however, the influence of any nonlinear process would be minimised, as tracer kinetics were determined in the presence of the same cold dose of prednisolone.

The results of our studies using plasma, urine, and faecial samples from volunteers suggest an almost complete absorption of prednisolone from the normal intestine, an observation similar to those reported by others using different techniques. ${ }^{10}$ In patients with Crohn's disease, however, absorption of prednisolone was incomplete and extremely variable, as judged by urinary excretion, faecal loss, and plasma appearance of radioactivity after oral ingestion of labelled prednisolone. In those subjects whose faecal losses were measured it was clear that these accounted for more than $50 \%$ of the differences between urinary excretion and oral intake, suggesting that the lower urinary excretion in Crohn's patients did, indeed, indicate malabsorption. Furthermore, there was a wide scatter of urinary recoveries and areas under the plasma concentration-time curves after oral administration in Crohn's patients, suggesting that variable absorption might be one important factor in the variable responsiveness of the disease.

Using a radioimmunoassay for prednisolone in plasma, Tanner et al ${ }^{11}$ did not show any malabsorption in a group of nine patients with Crohn's disease compared with normal volunteers. These results are at variance with ours, although comparisons are difficult because no intravenous standard was used in their study. It is worth noting, however, that the variability in plasma concentration-time profiles in their Crohn's patients was considerably greater than in the normal volunteers.

The reason for apparent malabsorption in Crohn's patients is unclear, as prednisolone is lipid soluble and should be readily absorbed by passive diffusion. A reduced surface area of the diseased, or resected, intestine, rapid transit, dispersion in bulky unabsorbed luminal contents, or changes in mucosal drug metabolising enzymes may all contribute to malabsorption. Whatever the cause, it seems reasonable to suggest that this variable absorption should be taken into account when assessing responses in patients with Crohn's disease.

This study was supported by a grant from the North West Regional Health Authority. The authors would like to acknowledge the technical assistance of K Boland, E Callaghan, and M Poulton.

\section{References}

1 Swartz SL, Dluhy RG. Corticosteroids: clinical pharmacology and therapeutic use. Drugs 1978; 16: 238-55.

2 Best WR, Bectel JM, Singleton JW, Kern F, Jr. Development of a Crohn's disease activity index. Gastroenterology 1976; 70: 439-40. 
3 Pickup ME. Clinical pharmacokinetics of intravenous and oral prednisolone. Clin Pharmacokinet 1979: 4: 111-28.

4 Gambertoglio JG. Amend WJC. Benet LZ. Pharmacokinetics and bioavailability of prednisone and prednisolone in healthy volunteers and patients: a review. J Pharmacokinet Biopharm 1980: 28: 1-52.

5 Pickup ME, Lowe JR, Leatham PA. Rhind VM. Wright V. Downie WW. Dose dependent pharmacokinetics of prednisolone. Eur J Clin Pharmacol 1977: 12: 21.3-9.

6 Tanner A. Bochner F. Caffin J. Halliday J. Powell L. Dose-dependent prednisolone kinetics. Clin Pharmacol Ther 1979: 25: 571-8.

7 Rocci ML. Johnson NF, Jusko WJ. Serum protein binding of prednisolone in four species. J Pharm Sci
198(): 69: 977-8.

8 Rose JQ, Yurchak AM. Jusko WJ. Bioavailability and disposition of prednisone and prednisolone from prednisone tablets. Biopharm Drug Dispos 1980; 1: 247-58.

9 Rocci ML. Szefler SJ. Acara M. Jusko WJ. Prednisolone metabolism and excretion in the isolated perfused rat kidney. Drug Metab Dispos 1981; 9: 177-82.

10 Al-Habet S. Rogers HJ. Pharmacokinetics of intravenous and oral prednisolone. Br J Clin Pharmacol 1980; 10: 503-8.

11 Tanner AR, Halliday JW. Powell L. Serum prednisolone levels in Crohn's disease and coeliac disease following oral prednisolone administration. Digestion 1981: $21: 310-5$. 